\title{
ALMOST GLOBAL ATTRACTION IN PLANAR SYSTEMS
}

\author{
Pablo Monzón * \\ * Instituto de Ingeniería Eléctrica, Facultad de Ingeniería, \\ Universidad de la República, Uruguay. \\ e-mail: monzon@fing.edu.uy
}

\begin{abstract}
In this work we present a result relating the recent ideas of almost global stability and density functions with the classical Poincaré-Bendixson Theory for planar systems.
\end{abstract}

Keywords: Nonlinear systems, Poincaré-Bendixson, almost global stability, planar systems.

\section{INTRODUCTION}

The almost global stability of dynamical systems is a concept weaker than global asymptotical stability but that can fit well in nonlinear control applications, specially when it is combined with local asymptotical stability. The concept and a sufficient condition for almost global stability were stated in the year 2001 by Anders Rantzer (Rantzer, 2001b) as a dual Lyapunov Method and has opened a new research direction in the nonlinear control field for both analysis and synthesis. The main result in (Rantzer, 2001b) is based on the existence of a density function, a kind of a dual of a Lyapunov function, that allows us to measure the growth of given sets along the flow.

In this work we explore the conditions that the existence of non-preserving measures impose to the behavior of two dimensional vector fields, blending the ideas of almost global attraction with the classical result of Poincaré-Bendixson. We think that this ideas can help to understand density functions and almost global stability.

In Section 2 we recall the basic definitions of almost global stability and density functions and the results between both concepts. We briefly introduce also the idea of monotone measure. After that we state and prove the main result for planar systems. Finally we present a counterexample in dimension three and some conclusions.

\section{PRELIMINARIES}

In this Section we introduce the works of Anders Rantzer reported in several papers appeared in the last years. We said that the origin is an almost global attractor (a.g.s.) if the complement of the set of points that are attracted to the origin has zero Lebesgue measure. For $x_{0} \in \mathcal{R}^{n}$, let $f^{t}\left(x_{0}\right)$ denote the time $t$ of the trajectory that starts at $x_{0}$. Then the system is a.g.s. if the set

$$
\left\{x \in \mathcal{R}^{n} \mid \lim _{t \rightarrow+\infty} f^{t}(x) \neq 0\right\}
$$

has zero Lebesgue measure. This concept of stability is weaker than the classical global asymptotic stability (g.a.s.) but can complement well the local asymptotical stability (l.a.s.) property. The key contribution of (Rantzer, 2001b) was the introduction of a particular kind of functions that for a.g.s. systems play a role similar to the Lyapunov functions for asymptotically stable systems: the density functions. Given a dynamical system $\dot{x}=$ $f(x)$, a density function for this system is a scalar function $\rho: \mathcal{R}^{n} \backslash\{0\} \rightarrow[0,+\infty)$, of class $C^{1}$, 
integrable outside of a ball centered at the origin, and such that the following divergence condition is satisfied

$$
\nabla \cdot(\rho f)(x)>0 \text { almost everywhere (a.e.) }
$$

A density function gives us a system-related way of measure sets in $\mathcal{R}^{n}$. The main result in (Rantzer, 2001b) says that the existence of a density function implies the almost global stability of the origin. Some steps has been done in order to prove that the condition is also necessary (Monzon, 2003; Rantzer, 2002). Some works have explored the analysis and synthesis of nonlinear control systems (Parrilo, 2000; Ceragioli, 2001; Rantzer, 2001a; Angeli, 2002; Angeli, 2003).

The existence of a density function implies the existence of a Borel measure $\mu$ for the state space that grows along the trajectories: i.e., for every non-zero $\mu$-measure set $Z$ and every positive time $t$,

$$
\mu\left[f^{t}(Z)\right]>\mu(Z)
$$

The measure $\mu$ also satisfies that $\mu\left[B^{c}(0, \epsilon)\right]<\infty$ for every $\epsilon>0$. We refer to that as a monotone measure bounded at infinity. The existence of monotone measures is a necessary and a sufficient condition for some kind of almost global stable systems, as was studied in (Monzon, 2003b). In the next sections we will assume the existence of a measure with some particular properties. In some cases, the existence of a monotone measure, or the existence of a density function, put us in that situation.

\section{THE MAIN RESULT}

We present here a result for two dimensional spaces that combines non-preserving measures with the Poincaré-Bendixson Theory. First of all we recall the definition of the $\omega$ and $\alpha$ limit sets for a given trajectory.

Definition 3.1. For $x \in \mathcal{R}^{n}$, we define $\omega$-limit of $x$ as the set

$$
\begin{array}{r}
\omega(x)=\left\{y \in \mathcal{R}^{n} \mid \exists\left\{t_{n}\right\} \text { with } \lim _{n} t_{n}=+\infty,\right. \\
\left.\lim _{n \rightarrow+\infty} f^{t_{n}}(x)=y\right\}
\end{array}
$$

The $\alpha$-limit is defined in the same way with $t_{n} \rightarrow$ $-\infty$.

It can be proved that if the trajectory $\left\{f^{t}(x)\right\}$ is bounded for $t \rightarrow \pm \infty, \omega(x)(\alpha(x))$ is a non-empty, compact, connected and invariant set (Khalil,
1996) and we can talk of the $\omega(\alpha)$-limit of the whole trajectory through $x$.

Theorem 3.1. Consider the system

$$
\dot{x}=f(x)
$$

where $f \in C^{1}\left(\mathcal{R}^{2}, \mathcal{R}^{2}\right)$. Assume that there is a finite number of fixed points in any compact set of $\mathcal{R}^{2}$. Suppose there exists a measure $\mu \ll m$, with $m$ the Lebesgue measure, satisfying that for every bounded and measurable set $Y$, with $0<\mu(Y)<\infty$, there exists $t \neq 0$ such that

$$
\mu\left[f^{t}(Y)\right] \neq \mu(Y)
$$

Then, almost global stability (a.g.s.) of the origin implies local asymptotical stability (l.a.s.).

\section{Proof:}

The Poincaré-Bendixson Theorem (Khalil, 1996) states that for a given point $x \in \mathcal{R}^{2}$ whose positive orbit is bounded, $\omega(x)$ can be only

(1) a singular point

(2) a closed orbit

(3) singular points $p_{1}, p_{2}, \ldots, p_{n}$ and regular orbits $\gamma$ such that $\alpha(\gamma)=p_{i}, \omega(\gamma)=p_{j}$, $i, j=1, \ldots, n$. (a)

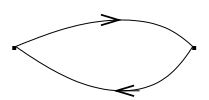

(c)

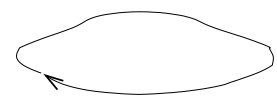

Figure 1. Possible structures for a non empty $\omega$ limit set.

The same result follows for the $\alpha$-limit set (Khalil, 1996). Typical $\omega$-limit ( $\alpha$-limit) possible sets for a point $x$ are shown in figure 1 . The hypothesis (2) about $\mu$ implies that the only possible situation for a non empty $\omega$-limit ( $\alpha$-limit) set is a single fixed point, case (d) in figure 1 , since cases (a), (b) and (c) contains an invariant non-zero Lebesgue measure set. This will be an important fact.

We will prove the thesis by contradiction. Suppose that the origin is not a locally asymptotically stable fixed point. Then, there is an $\epsilon>0$, small enough to ensure that $x=0$ is the only singular point inside the open ball $B(0, \epsilon)$, such that for every non-zero $n \in \mathcal{N}$ we can find a $x_{n} \in \mathcal{R}^{2}$ with

$$
\left\|x_{n}\right\|<\frac{1}{n}, \sup _{t \geq 0}\left\{\left\|f^{t}\left(x_{n}\right)\right\|\right\}>\epsilon
$$

as in figure 2. Define $z_{n}$ as the first intersection of the trajectory $\left\{f^{t}\left(x_{n}\right)\right\}$ with the sphere $S(0, \epsilon)$. Then we obtain a sequence $\left\{z_{n}\right\}_{n \in \mathcal{N}}$ of points 


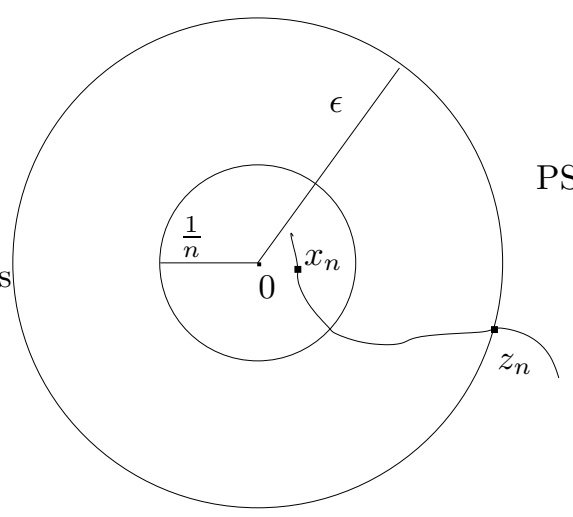

Figure 2. Finding the sequence $\left\{x_{n}\right\}$.

with norm equal to $\epsilon$, whose trajectories to the past come close to the origin. Since $S(0, \epsilon)$ is a compact set, we can find a sub-sequence, which we still call $\left\{z_{n}\right\}$, converging to a point $z \in S(0, \epsilon)$. We affirm that

$$
\alpha(z)=\{0\}
$$

If it is not the case, there is a positive real $a$ such that the trajectory never goes inside the ball $B(0, a)$. Then, since $x=0$ is the only singular point in $B(0, \epsilon)$, the trajectory $\left\{f^{t}(z)\right\}$ leaves the ball $B(0, \epsilon)$. The situation is shown in figure 3 .

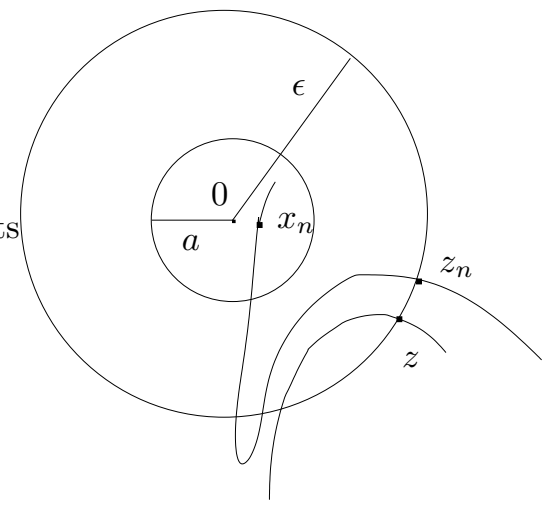

Figure 3. Case: $\alpha(z) \neq\{0\}$.

Then every trajectory starting close enough to $z$ will accompany $\left\{f^{t}(z)\right\}$ to the past out of the ball and there exists a non-zero natural $N_{1}$ such that for every $n>N_{1}$, the trajectory $\left\{f^{t}\left(z_{n}\right)\right\}$ leaves the ball for some negative $t$. On the other hand, there is a non-zero natural $N_{2}$ such that for every $n>N_{2}$

$$
\inf _{t \leq 0}\left\{\| f^{t}\left(z_{n} \|\right\}<\frac{1}{n}\right.
$$

Then, for every $n>\max \left\{N_{1}, N_{2}\right\}$, the negative trajectory through $z$ leaves $B(0, \epsilon)$ before it gets close to the origin, but this can not occur since $z_{n}$ was defined in a way such that the piece of trajectory from $x_{n}$ to $z_{n}$ is totally in the inside of the closed ball $\overline{B(0, \epsilon)}$. Then $\alpha(z)=\{0\}$.

Now consider a transversal section to the trajectory through $z$. On this section, we can find a

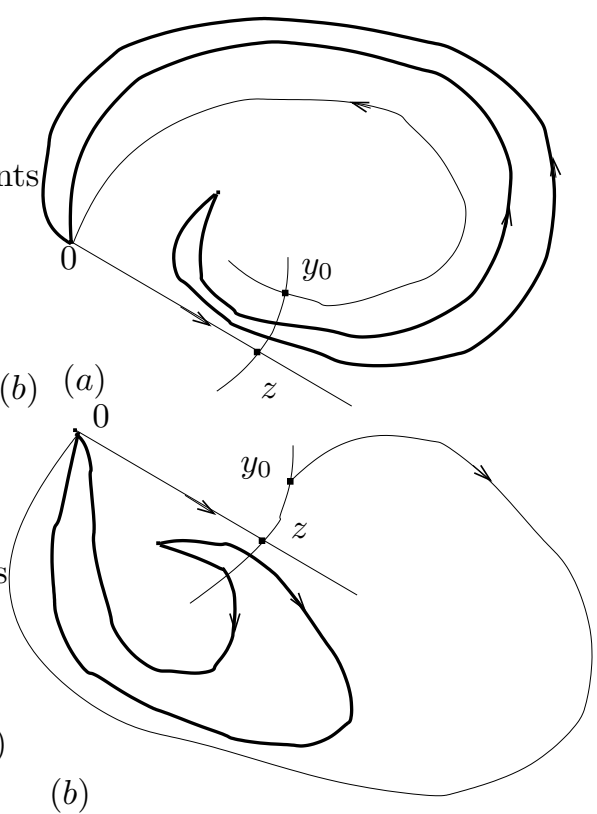

Figure 4. Non zero measure invariant sets.

point $y_{0}$, arbitrarily close to $z$, whose $\omega$-limit is the origin, since this kind of trajectories form a dense set due to the a.g.s assumption. Then, as in the Poincaré-Bendixson Theorem, we can construct a closed path with the negative trajectory through $z$, a piece of the transversal section and the positive trajectory through $y_{0}$. This path limits a closed region of the plane, with a finite number of fixed points inside it. The first situation we can have is the one shown in figure 4-(a). On the transversal section, we can find two points whose $\omega$-limit sets are the origin and their $\alpha$-limit sets are some singular point (could be other than the origin). The trajectories through these points are like the bold ones in figure 4-(a). The other case is shown in 4-(b) and the result is the same of the case (a). In both situations, the sets limited by the bold trajectories are invariant and have non zero Lebesgue measure. This is an absurd and then the origin is a locally stable fixed point.

\section{Observations:}

First note that if the measure $\mu$ is monotone, then

the condition (2) is fulfilled.

The almost global stability assumption can be relaxed. It is enough to ask that the set of trajectories attracted by the origin is dense in the plane; that is, given a point $x \in \mathcal{R}^{2}$ and $\delta>0$, there is a point $y \in \mathcal{R}^{2}$ such that

$$
\|x-y\|<\delta \quad \text { and } \quad \lim _{t \rightarrow+\infty} f^{t}(y)=0
$$

We apply the previous result in order to characterize the behavior at infinity of an almost globally stable system. 
Theorem 3.2. Consider the complete nonlinear system $\dot{x}=f(x)$ with $f \in C^{1}\left(\mathcal{R}^{2}, \mathcal{R}^{2}\right)$. Assume that the set $f^{-1}(\{0\})$ is finite in $\mathcal{R}^{2}$ and that there is a monotone measure $\mu$ bounded at infinity. If the set

$$
A=\left\{x \in \mathcal{R}^{2} \mid \lim _{t \rightarrow+\infty}\left\|f^{-t}(x)\right\|=+\infty\right\}
$$

is dense in $\mathcal{R}^{2}$ then the $\infty$ is a locally asymptotically stable point to the past.

\section{Proof:}

We have to show that given an arbitrary positive number $M$, there is a positive number $K$, depending on $M$, such that

$$
\text { if }\|x\|>K \Rightarrow\left\|f^{-t}(x)\right\|>M \quad \forall t \geq 0
$$

and that $K$ can be chosen just that $\left\|f^{-t}(x)\right\| \rightarrow$ $+\infty$.

Instead of that, we will compactify the plane using the stereographic projection in order to work on the compact Riemann sphere. Doing this, we obtain a dynamical system on the sphere with an a.g.s. equilibrium point at the south pole $S$ (corresponding to the origin of the plane) and an equilibrium point at the north pole $N$ (corresponding to the infinity of the plane). We know that $N$ attracts a dense set of trajectories to the past and that we can define a Borel measure $\mu$ over the sphere in a way that given any non zero Lebesgue measure neighborhood $Y$ of $N$ with $S \notin \bar{Y}$, it verifies $0<\mu(Y)<\infty$ and for every $t>0$,

$$
\mu\left[f^{t}(Y)\right]>\mu(Y)
$$

Then we consider the reversed system over the sphere

$$
\dot{x}=-f(x)
$$

and we obtain that $N$ attracts a dense set of trajectories. We can reconstruct the proof of Theorem 3.1, denying the Thesis and getting the existence of the bold trajectories of figure 4 . If the set $A$ enclosed by this curves has finite measure $\mu$ we get an absurd, just as in the previous proof. So, the question we must answer is if $S \in \bar{A}$. But if it was the case, $S$ would be the $\alpha$-limit of the bold trajectories and then $S$ could not attract almost all the trajectories of the original system. In order to see this, consider again the closed path constructed with the negative trajectory of $z$, the positive trajectory of $y_{0}$ and a piece of the transversal section through $z$. We draw again the picture in figure 5 .

Then all the trajectories started outside this closed path must enter it to reach $S$ and these can be done only through the piece of transversal section, which can be made arbitrarily small because

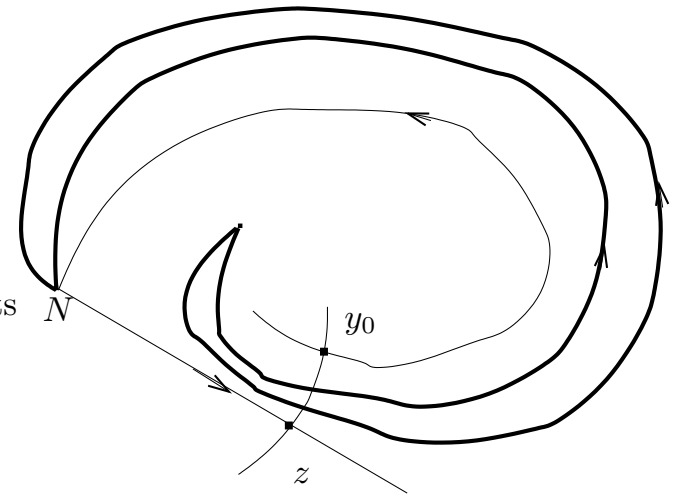

Figure 5. Situation on Theorem 3.2.

of the dense assumption on the set of trajectories attracted by the north pole $N$ to the past.

The counter-reciprocal version of the previous Theorem is very interesting.

Corollary 3.2.1. Consider the nonlinear system $\dot{x}=f(x)$ with $f \in C^{1}\left(\mathcal{R}^{2}, \mathcal{R}^{2}\right)$. Assume that the set $f^{-1}(\{0\})$ is finite in $\mathcal{R}^{2}$ and that there is a monotone Borel measure $\mu$ bounded at infinity. If there is at least one trajectory that goes to infinity to the future, then the set of trajectories that go to infinity to the past is not dense in $\mathcal{R}^{2}$.

Example 3.1. (Rantzer, 2001b) Consider planar the system

$$
\left[\begin{array}{c}
\dot{x}_{1} \\
\dot{x}_{2}
\end{array}\right]=\left[\begin{array}{c}
-2 x_{1}+x_{1}^{2}-x_{2}^{2} \\
-6 x_{2}+2 x_{1} x_{2}
\end{array}\right]
$$

It has four equilibria at $(0,0),(2,0)$ and $(3, \pm \sqrt{3})$. We note that the axis $\left\{x_{2}=0\right\}$ is an invariant set. Then, if we consider the initial condition $\left(x_{10}, 0\right)$ with $x_{10}>2$, we find out that the trajectory goes to infinity. Besides that, the system admits a density function

$$
\begin{gathered}
\rho\left(x_{1}, x_{2}\right)=\left[x_{1}^{2}+x_{2}^{2}\right]^{-2} \\
\nabla \cdot[\rho f]\left(x_{1}, x_{2}\right)=16 \cdot x_{2}^{2} \cdot\left[x_{1}^{2}+x_{2}^{2}\right]^{-4}
\end{gathered}
$$

Observe that the local stability of the origin and the existence of the monotone Borel measure prevent the existence of limit cycles. Then, we can conclude that there exist trajectories that do not go to infinity to the past and then they must go to another equilibrium point.

Of course the previous result of Example 3.1 could have been obtained through other ways. For example we can classify the equilibrium points and realize that the only divergent trajectory is 
the one we have found. Moreover, the trajectories that are not attracted by the origin are this one and the stable manifold of $(2,0)$, as can be seen in figure 6.

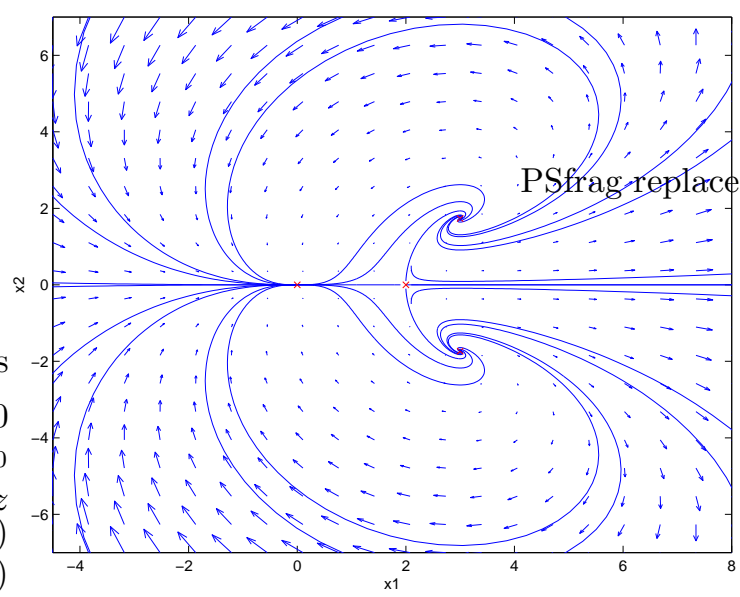

Figure 6. System of Example 3.1.

The main result of this section is deeply grounded on the topological consequences of the dimension 2 . The following example shows that in dimension 3 we can have a measure satisfying (2) but the origin can be a.g.s. and not locally stable.

Example 3.2. Consider the following dynamical system defined on $\mathcal{R}^{3}$ :

$$
\left\{\begin{array}{l}
\dot{x}=x^{2}-y^{2} \\
\dot{y}=2 x y \\
\dot{z}=-z
\end{array}\right.
$$

On the $z$ direction we have the decoupled dynamic

$$
z(t)=e^{-t} \cdot z_{0}
$$

and at the plane $z=0$ the dynamic has the phase portrait depicted in figure 7 . As can be proved analytically, the trajectories on $z=0$ are circumferences with the center on the line $x=0$. So the origin $(0,0,0)$ is an almost globally stable equilibrium point but not locally stable.

We will see that the Lebesgue measure $\lambda$ verifies (2). Consider a bounded nonzero measure set $C \subset \mathcal{R}^{3}$. It can be covered by a bounded rectangle $A \times B$, with $A \subset \mathcal{R}^{2}$ and $B \subset \mathcal{R}$ bounded Borel sets. It is clear that if we denote by $F$ the field on $\mathcal{R}^{3}$, we have

$$
\lambda\left[F^{t}(A \times B)\right]=m\left[f^{t}(A)\right] \cdot e^{-t}|B|
$$

where $|B|$ stands for the length of the interval $B$ and $m$ denotes the Lebesgue measure on $\mathcal{R}^{2}$. The numbers $m\left[f^{t}(A)\right]$ are bounded since almost all the trajectories converge to the $(0,0)$ in the plane $z=0$. Then there is a time $t$ big enough such that

$$
\lambda\left[F^{t}(A \times B)\right]<\lambda[A \times B]
$$

and $\lambda$ verifies (2).

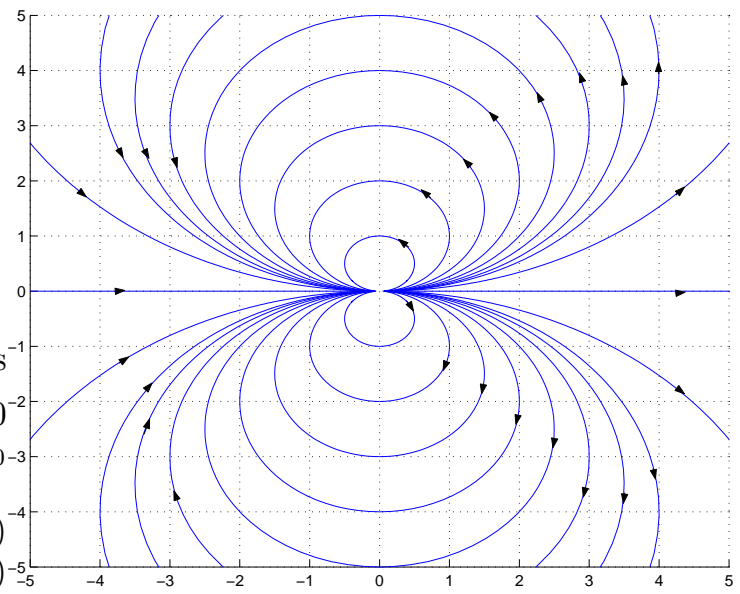

Figure 7. System of Example 3.2 on $z=0$ (we have taken $\rho_{0}=4$ ).

\section{CONCLUSIONS}

We have presented a result for planar systems that states a relationship between the new concepts of almost global stability and density functions and the classical Theorem of Poincaré- Bendixson. We have also shown a counter-example in $\mathcal{R}^{3}$ in order to emphasize that the result is based on the topological structure of two dimensional systems.

\section{ACKNOWLEDGEMENTS}

I want to specially thank Professor Jorge Lewowicz for his time, dedication and advice.

\section{REFERENCES}

Angeli, D. (2002). An almost global notion of input-to-state stability. In: Conference on Decision and Control. IEEE CSS. Las Vegas, USA.

Angeli, D. (2003). Some remarks on density functions for dual lyapunov methods. In: Conference on Decision and Control. IEEE CSS. Hawaii, USA.

Ceragioli, A. Rantzer; F. (2001). Smooth blending of nonlinear controllers using density functions. European Control Conference. Porto.

Khalil, Hassan (1996). Nonlinear Systems. Prentice-Hall.

Monzon, P. (2003). On necessary conditions for almost global stability. IEEE Trans. Automatic Control 48(4), 631-634.

Monzon, P. (2003b). Monotone measures and almost global satability of dynamical systems. Pre-Publicaciones Matemticas de la UdelaR, $2003 / 73$. 
Parrilo, A. Rantzer; P. (2000). On convexity in stabilization of nonlinear systems. In: IEEE Conference on Decision and Control. Sidney.

Rantzer, Anders (2001a). Almost global stability of phase-locked loops.. Technical report. Lund Institute of Technology.

Rantzer, Anders (2001b). A dual to Lyapunov's stability theorem. Systems and Control Letters 42(3), 161-168.

Rantzer, Anders (2002). A converse theorem for density functions. IEEE. 